Relations industrielles

Industrial Relations

\title{
Leadership, Psychology and Organizational Behavior, by Bernard M. Bass, Harper \& Brothers, New York, 548 pp.
}

\section{C.-R. Giroux}

Volume 15, numéro 2, avril 1960

URI : https://id.erudit.org/iderudit/1022046ar

DOI : https://doi.org/10.7202/1022046ar

Aller au sommaire du numéro

Éditeur(s)

Département des relations industrielles de l’Université Laval

ISSN

0034-379X (imprimé)

1703-8138 (numérique)

Découvrir la revue

Citer ce compte rendu

Giroux, C.-R. (1960). Compte rendu de [Leadership, Psychology and Organizational Behavior, by Bernard M. Bass, Harper \& Brothers, New York, 548 pp.] Relations industrielles / Industrial Relations, 15(2), 281-283.

https://doi.org/10.7202/1022046ar

Tous droits réservés (C Département des relations industrielles de l’Université Laval, 1960
Ce document est protégé par la loi sur le droit d'auteur. L’utilisation des services d'Érudit (y compris la reproduction) est assujettie à sa politique d'utilisation que vous pouvez consulter en ligne.

https://apropos.erudit.org/fr/usagers/politique-dutilisation/ 
uses of the communication process within the business setting ». Cette optique limite le public auquel il s'adresse.

L'aspect le plus innovateur consiste dans la façon dont l'auteur conçoit les communications et la place qu'il leur accorde dans le travail de l'administrateur. Il en fait la fonction essentielle.

« Today's developing concept of leadership, exercised largely by persuasion rather than by command, places a great new premium on communication. Three of the elements of a manager's work, planning, organizing and measuring, are heavily dependent on employee communication: the fourth category, integration, is almost synonymous with communication 》 (p. 30). Plus loin, il ajoute: «Communication, is not a constricted concept of getting work performed by people through listening and talking and writing to them. Neither is it a concept of manipulating people through hypnotic semantics. Rather, communication is a process which both affects and is affected by the deeds and attitudes of management, on one hand, and the employees and public on the other. It is essentially an interpretive process. This implies that the successful business communicator - like the successful translator of languages must not only know the languages of both the senders and the receivers, but must also have a sympathetic understanding of the backgrounds, the attitudes and aspirations of both. Communication, then, is a broad conception equaling, in its magnitude and importance to the business, such other accepted or upcoming practices as automation, decentralization or operations research and synthesis 》 (p. 36).

C'est une conception très compréhensive de la fonction communication. L'auteur ne la perd jamais de vue dans les techniques qu'il propose. Il y revient même à plusieurs reprises pour rappeler que ce procédé d'interprétation est dynamique, qu'il est instrumental et non une fin en lui-même. "Communication is a mean - and a very effective mean - for the solution of managerial problems and for the attainment of managerial objectives » (p. 235).

Mais l'auteur amène, élabore, décrit des techniques et des principes avec parfois une confiance naïve dans leur efficacité ou leur capacité de solutionner à peu près tous les problèmes de la direction avec les employés, les syndicats et la communauté en général. Nul doute que ce livre peut aider à améliorer la productivité et la satisfaction au travail, à créer un climat plus réceptif devant l'innovation et à établir des conditions qui favorisent la compréhension mutuelle. Mais nous tenons à mettre le lecteur en garde contre l'impression qui se dégage à certains moments à l'effet qu'une recette éprouvée dans un certain milieu sera nécessairement valable ailleurs. Au contraire, ces recettes ne sont pas des arguments d'autorité, mais des exemples de ce qu'un peu d'imagination et de bonne volonté peuvent réaliser.

En résumé, ce livre comporte cinq parties d'inégale valeur ou importance mais d'un intérêt réel. La première partie porte sur la nature des communications; la seconde illustre comment l'administrateur doit utiliser les communications pour améliorer ses relations avec les employés, le syndicat et la communauté; la troisième traite $\mathrm{du}$ rôle du fonctionnaire en position d'autorité, spécialement du contremaître: la quatrième déorit un nombre imposant de techniques. principes et moyens de communications (orales - écrites - vers le haut, vers le bas, etc.); la cinquième enfin applique l'usage des communications à certaines fins spécifiques, telles que: les changements, les périodes de crises, les grèves, etc.

Somme tcute, un assez bon volume qui fait une synthèse de l'expérience actuelle et qui s'avérera utile pour celui qui est engagé dans l'administration.

Ls-Marie Tremblay

\section{Leadership, Psychology and Organiza- tional Behavior, by Bernard M. Bass, Harper \& Brothers, New York, 548 pp.}

Depuis quelques années l'on essaie de découvrir et de définir le meneur du groupe - «leader» - non plus par des traits individuels ou selon la théorie du grand homme mais surtout en fonction de la dynamique de groupe. En effet, ce qui justifie l'attribut de mereur c'est l'influence exercée sur le groupe. Plusieurs travaux ont été publiés récemment sur le développement 
du groupe et sur la structure de l'organisation; parmi les plus importants notons ceux de Bellows, Bonner et Haire. Toutefois cette récente publication de Bass les surpasse tous de beaucoup.

Depuis longtemps déjà, Bass est reconnu comme l'expert de la dynamique du groupe démocratique - « Leaderless Group Discussion Method - LDG ». Cette méthode lui a servi à formuler et à vérifier de nombreuses hypothèses. Dans son présent ouvrage, Bass s'est fixé un objectif précis. Pour l'atteindre, il faut plus qu'un niveau d'aspiration élevé. Il faut procéder de façon méthodique, analyser tout ce qui se rapporte au sujet, faire une synthèse des résultats expérimentaux, en extraire des hypothèses et des postulats et enfin tenter d'élaborer une théorie unifiée. C'est bien l'objectif que s'est tracé Bass: développer une théorie de la dynamique du \& leader » et du groupe. Théoriser sur le comportement du groupe! Mais n'est-ce pas un peu téméraire? D'abord il faut consulter toute une quantité énorme de travaux sur tous les types de groupes possibles et impossibles; depuis les groupes de deux membres, les groupes ethniques, les groupes du «Air Defense Early Warning System》, jusqu'aux masses. Les frontières de ce domaine sont indéterminées et changeantes. Cette gamme variée de contenu et de méthodes d'étude est caractérisée par l'élément hétéro-disciplinaire. En effet, tous s'intéressent à la dynamique de groupe, psychologues, sociologues, éoonomistes, mathématiciens, ingénieurs, philosophes, etc. Les concepts utilisés varient avec les écoles et les disciplines. Les recherches ne répondent pas toutes aux exigences rigoureuses des conditions expérimentales. Malgré un tel handicap, Bass réussit à se retrouver dans ce labyrinthe. En plus de manifester sa grande culture, (il est aussi à l'aise dans Esope, Platon, Aristote que dans les auteurs modernes), il fait preuve d'une grande compétence professionnelle. En effet, il revoit à peu près tout ce qui s'est publié sur le sujet. La bibliographie comprend 1155 publications différentes.

Dans la théorie qu'il offre au lecteur, Bass assume que quelques facteurs tels que la possibilité d'interaction, la motivation, le rang du meneur et les caractéristiques du groupe suffisent à rendre compte de la variété du \&leadership > et du comportement du groupe. En déterminant les limitations, et les demandes fonctionnelles imposées au «leader » dans des situations déterminées, et après, en déterminant les caractéristiques personnelles des individus les plus aptes à se plier à ces demandes et à ces limitations, l'on sera en mesure de prédire le succès possible et l'efficacité de chaque candidat au poste de meneur dans une situation spécifique, parce que l'on connaîtra alors les qualités spécifiques de la situation et de chaque candidat.

Bass réserve tout un chapitre sur la nature et le but d'une théorie. Il décrit clainement sa philosophie de la recherche, i.e. le positivisme logique. La recherche est un processus de raisonmement qui se déroule sur deux plans, celui des faits observables et celui des concepts. A partir des faits le chercheur élabore et définit des concepts. Il induit des postulats à partir des relations établies entre les concepts. Des théorèmes sont déduits des postulats et servent à formuler des hypothèses qu'il faut alors vérifier expérimentalement. Bass suit exactement cette méthode de recherche dans l'exposé de sa théorie. Chaque chapitre débute par une citation philosophique soit d'Aristote, de Plutarque, de Machiavel, de Han Fu-Tse, etc., qui sert à exposer un ooncept. Puis l'auteur énonce les postulats et expose ses hypothèses. Ensuite, il résume toutes les expériences et les rechenches qui confirment ou infirment ses hypothèses. Les références sont nombreuses et bien choisies; toutefois, Bass ne commente pas la validité et la fiabilité des recherches utilisées dans son exposé. Sa définition du «leadership » se lit comme stiit: \& Leadership is the observed effort of one member to change other members' behavior by altering the motivation of the other members or by changing their habits \$.

Résumons maintenant la théorie de Bass. Elle débute par une considération du groupe en tant qu'agent de satisfaction pour une collectivité d'individus. Plus un groupe satisfait les besoins de ses membres plus il est efficace; un groupe sera d'autant plus attrayant que les. membres anticipent la satisfaction de leurs besoins. Il est intéressant de noter ici les motivations qui peuvent influencer l'interaction entre le < leader \$ et les membres du groupe. Trois motivations différentes oonditionnent cette 
interaction. Le eleader》 et les groupes peuvent être motivés soit à résoudre des problèmes «Task Oriented 》, soit à satisfaire leurs propres besoins personnels « Self Oriented ». Le «leader $\gg$ efficace sera celui qui pourra diriger le groupe vers une action créatrice dans la solution des problèmes communs. Par son comportement personnel il accondera aux membres de la reconnaissance pour leur performance et réduira ainsi les possibilités de punition.

Les membres qui auront expérimenté un certain succès avec un \&leader » auront tendance à suivre cette même personne dans l'avenir. De même, un «leader efficace aura plus de chances de réussir dans d'autres situations semblables et son succès passé l'amènera à essayer de conduire le groupe quand les circonstances l'exigeront. Les expé riences rapportées par Bass semblent indiquer que le membre le plus capable deviendra tout probablement le «leader $\gg$ du groupe. Si ce membre a l'habileté nécessaire pour résoudre les problèmes du groupe, il semble qu'il réussira à convaincre les membres à accepter son autorité. Il semble que certains membres en vertu de leur position ou de leurs qualités personnelles sont en mesure de récompenser ou de punir directement les autres membres du groupe. Un individu jouissant d'un rang élevé, qui lui permet de récompenser ou de désapprouver l'action du groupe, peut forcer le groupe à atteindre un but déterminé. Nous faisons face à ce moment là à une autorité qui devient facilement absolue et parfois même arbitraire. Si toutefois la récompense ou la punition est déterminée par le groupe ou est utilisée prudemment, le «leader 》, même jouissant d'une autorité hiérarchique exercera alons une action permissive. Notons toutefois que la coercion et la permission requièrent toutes deux le pouvoir délégué par une autorité hiéranchique. Dans un groupe démocratique, une telle autorité étant accordée au «leader » par les membres du groupe, il ne peut pas utiliser la coercion. Nous constatons donc que le conformisme ne peut exister qu'en fonction d'une interaction basée sur une hiérarchie bien structurée. Le prestige attaché à une position, et l'estime individuel, sont tous deux source d'habileté et de pouvoir. Quand le prestige et l'estime ne concordent pas les conflits surgissent facilement. Les deux dernières parties du volume traitent de divers attributs du groupe: le nombre des membres, la proximité entre eux, l'estime mutuel, les communications, la familiarité, l'homogénéité et les autres variables qui peuvent augmenter la possibilité d'interaction entre les membres. Tout changement dans la possibilité d'interaction affecte directement l'efficacité du groupe. Finalement l'auteur indique diverses méthodes pour augmenter l'efficacité d'un groupe.

La plupart des énoncés précédents. sont corroborés par des résultats expérimentaux. Toutefois, plusieurs restent encore à être démontrés de façon pratique.

Bass n'a pas la prétention d'élaborer une théorie définitive. Comme il le dit lui-même: «Paradoxically, a satisfactory theory begins to be modified almost as soon as it is cncluded: an inadequate one more often lasts unchanged for centuries. The goal of simplicity is congruent with the purpose of theorybuilding: to promote understanding $>$. Ceci se rapproche du fameux mot de J.J. Thomas: \& A theory is a policy rather than a creed $\gg$.

Cette oeuvre de Bass nous permet d'entrevoir les motivations en jeu dans la dynamique du groupe. Maintenant, il nous reste à tout mettre en pratique.

Cette brillante synthèse de Bass va certainement stimuler la recherche sur la dynamique et la structure des groupes.

$$
\text { C.-R. Giroux }
$$

Leadership Dynamics and the TradeUnion Leader. Lois MacDonald and Associates. N.Y. New York University Press, $1959,150 \mathrm{pp}$.

La croissance phénoménale des unions ouvrières depuis environ quinze ans a fait surgir une foule de problèmes d'interrelations de groupes et de nombreux conflits d'autorité. Une organisation interne inadéquate, l'incompétence et l'incompréhension des représentants syndicaux furent souvent à l'origine de frictions non seulement entre le capital et lo travail mais entre les membres eux- 